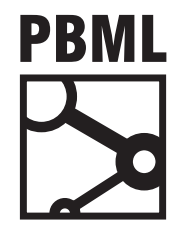

The Prague Bulletin of Mathematical Linguistics

NUMBER 109 OCTOBER 2017 15-28

\title{
NMTPY: A Flexible Toolkit for Advanced Neural Machine Translation Systems
}

\author{
Ozan Caglayan, Mercedes García-Martínez, Adrien Bardet, Walid Aransa, \\ Fethi Bougares, Loïc Barrault
}

Laboratoire d'Informatique de I'Université du Maine (LIUM)

\begin{abstract}
In this paper, we present nmtpy, a flexible Python toolkit based on Theano for training Neural Machine Translation and other neural sequence-to-sequence architectures. nmtpy decouples the specification of a network from the training and inference utilities to simplify the addition of a new architecture and reduce the amount of boilerplate code to be written. nmtpy has been used for LIUM's top-ranked submissions to WMT Multimodal Machine Translation and News Translation tasks in 2016 and 2017.
\end{abstract}

\section{Introduction}

nmtpy is a refactored, extended and Python 3 only version of dl4mt-tutorial - , a Theano (Theano Development Team, 2016) implementation of attentive Neural Machine Translation (NMT) (Bahdanau et al., 2014). The development of nmtpy project which has been open-sourced 2 under MIT license in March 2017, started in March 2016 as an effort to adapt dl4mt-tutorial to multimodal translation models. nmtpy has now become a powerful toolkit where adding a new model is as simple as deriving from an abstract base class, implementing a set of its methods and writing a custom data iterator if necessary. The training and inference utilities are as model-agnostic

\footnotetext{
${ }^{1}$ https://github.com/nyu-dl/dl4mt-tutorial

${ }^{2}$ https://github.com/lium- lst/nmtpy
} 
as possible allowing one to use them for different sequence generation networks such as multimodal NMT and image captioning to name a few.

Other prominent toolkits in the field are OpenNMT (Klein et al., 2017), Neural Monkey (Helcl and Libovický, 2017) and Nematus (Sennrich et al., 2017). While nmtpy and Nematus share the same dl4mt-tutorial codebase, the flexibility and the rich set of architectures (Section 3) are what differentiate our toolkit from Nematus. Both OpenNMT and Nematus are solely focused on translation by providing feature-rich but monolithic NMT implementations. Neural Monkey which is based on TensorFlow (Abadi et al., 2016), provides a more generic sequence-to-sequence learning framework similar to nmtpy.

\section{Design}

In this section we first give an overview of a typical NMT training session in nmtpy and the design of the translation utility nmt-translate. We then describe the configuration file format, explain how to define new architectures and finally introduce the basic deep learning elements and techniques provided by nmtpy. A more detailed tutorial about training an NMT model is available on Github $\underline{3}$.

\subsection{Training}

A training experiment (Figure 1) is launched by providing an INI-style experiment configuration file to $n m t$-train (Listing 1). nmt-train then automatically selects a free GPU, sets the seed for NumPy and Theano random number generators, constructs an informative filename for log files and model checkpoints and finally instantiates a Python object of type "model_type" given through the configuration file. The tasks of data loading, weight initialization and graph construction are all delegated to this model instance.

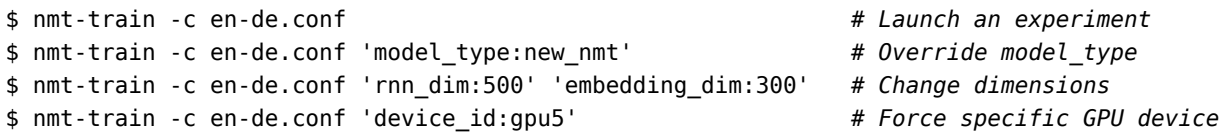

Listing 1. Example usages of nmt-train.

During training, nmt-train consumes mini-batches of data from the model's iterator and performs forward/backward passes along with the weight updates. Translation performance on a held-out corpus is periodically evaluated in order to early-stop the training process to avoid overfitting. These periodic evaluations are realized by calling nmt-translate which performs beam-search, computes metrics and returns them back to nmt-train.

\footnotetext{
${ }^{3}$ https://github.com/lium-lst/wmt17-mmt
} 


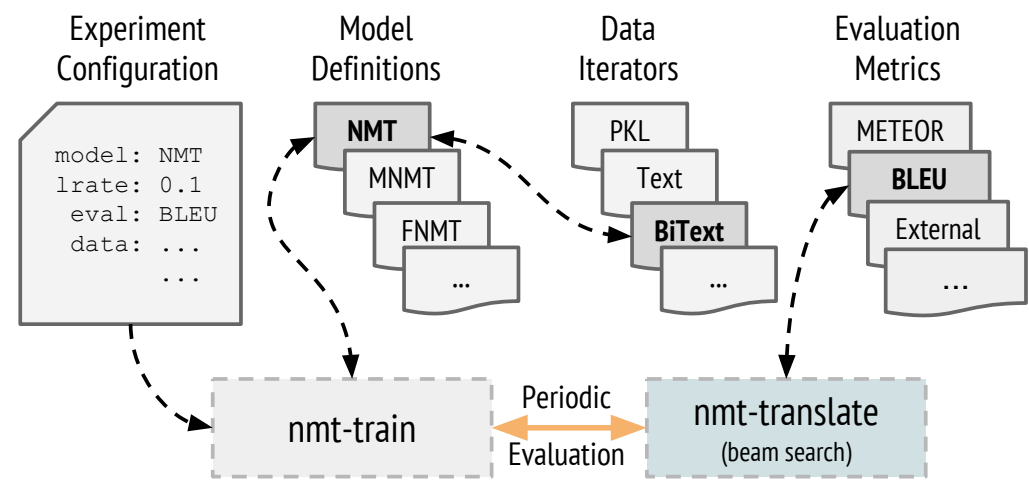

Figure 1. The workflow of a training experiment.

\subsection{Translation}

nmt-translate performs translation decoding using a beam-search implementation that supports single and ensemble decoding for both monomodal and multimodal translation models (Listing 2).

Since the number of CPUS in a single machine is $2 x-4 x$ higher than the number of GPUs and we mainly reserve the GPUs for training, nmt-translate makes use of CPU workers for maximum efficiency. More specifically, each worker receives a model instance (or instances when ensembling) and performs the beam-search on samples that it continuously fetches from a shared queue filled by the master process. One thing to note for parallel CPU decoding is that if the installed NumPy is linked against a BLAS implementation with threading support enabled (as in the case with Anaconda \& Intel MKL), each spawned process attempts to use all available threads in the machine leading to a resource conflict. In order for $n m t$-translate to benefit correctly from parallelism, the number of threads per process should thus be limited to one $\stackrel{4}{-}$. The impact of this setting and the overall decoding speed in terms of words/sec (wps) are reported in Table 1 for a medium-sized En $\rightarrow \operatorname{Tr}$ NMT with $\sim 10 \mathrm{M}$ parameters.

\# Decode on 30 CPUs with beam size 10, compute BLEU/METEOR

\$ nmt-translate -j 30 -b 10 -M bleu meteor -m model.npz -S val.bpe.en -R val.de -o out.de

\# Generate 50-best list with an ensemble of checkpoints

\$ nmt-translate -b 50 -N 50 -m model*npz -S val.tok.de -o out.tok.50best.de

Listing 2. Example usages of nmt-translate.

\footnotetext{
${ }^{4}$ This is achieved by setting $X \_N U M \_T H R E A D S=1$ environment variable where $X$ is one of OPENBLAS, OMP, MKL depending on the NumPy installation.
} 


\begin{tabular}{rrrrr}
\hline \# BLAS Threads & Tesla K40 & 4 CPU & 8 CPU & 16 CPU \\
\hline Default & $185 \mathrm{wps}$ & $25 \mathrm{wps}$ & $25 \mathrm{wps}$ & $25 \mathrm{wps}$ \\
Set to 1 & $185 \mathrm{wps}$ & $109 \mathrm{wps}$ & $198 \mathrm{wps}$ & $332 \mathrm{wps}$ \\
\hline
\end{tabular}

Table 1. Median beam-search speed over 3 runs with beam size 12: decoding on a single Tesla K40 GPU is roughly equivalent to using 8 CPUs (Intel Xeon E5-2687v3).

\subsection{Configuration}

Each nmtpy experiment is defined with an INI-style configuration file that has four mandatory sections, namely [training], [model], [model.dicts] and [model.data]. Each section may contain a number of options in key: value format where the value can be built-in Python data types like integer, float, boolean, string, list, etc. Paths starting with a tilde are automatically expanded to \$HOME folder.

The options defined in the [training] section are consumed by nmt-train while the ones in the [model.*] sections are automatically passed to the model instance (specifically, to its __init__ ( ) method) created by nmt-train. This allows one to add a new key : value option to the configuration file and access it automatically from the model instance.

Any option defined in the configuration file can be overridden through the command line by passing new key: value pair as the last argument to nmt-train (Listing 1). The common defaults defined in nmtpy/defaults. py are shortly described in Table $\underline{2}$. A complete configuration example is provided in Appendix A.

\subsection{Defining New Architectures}

A new architecture can be defined by creating a new file (i.e. my_amazing_nmt.py) under nmtpy/models, defining a new Model class derived from BaseModel and implementing 5 the set of methods detailed below:

- _ init_( ): Instantiates a model. Keyword arguments can be used to gather model specific options from the configuration file.

- init_params ( ): Initializes the layers and their weights.

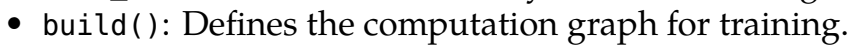

- build_sampler(): Defines the computation graph for beam-search. This is similar to build ( ) except two additional Theano functions.

- load_valid_data(): Loads the validation data for perplexity computation.

- load_data( ): Loads the training data.

\footnotetext{
${ }^{5}$ The NMT architecture defined in attention . py can generally be used as a skeleton code when developing new architectures.
} 


\subsection{Building Blocks}

Initialization Weight initialization is governed by the weight_init option and supports Xavier (Glorot and Bengio, 2010), He (He et al., 2015), orthogonal (Saxe et al., 2013) and random normal initializations.

Regularization An inverse-mode (the magnitudes are scaled during training instead of testing) dropout (Srivastava et al., 2014) can be applied over any tensor. $\mathrm{L}_{2}$ weight regularization with a scalar factor given by decay_c option is also provided.

\begin{tabular}{|c|c|c|}
\hline Option & Value & Description \\
\hline \multicolumn{3}{|l|}{ [training] options } \\
\hline init & None/<.npz file> & Pretrained checkpoint to initialize the weights. \\
\hline device_id & auto/cpu/gpu<int $>$ & Select training device automatically or manually. \\
\hline seed & 1234 & The seed for Theano and NumPy RNGs. \\
\hline clip_c & 5.0 & Gradient norm clipping threshold. \\
\hline decay_c & 0.0 & $\mathrm{~L}_{2}$ regularization factor. \\
\hline patience & 10 & Early-stopping patience. \\
\hline patience_delta & 0.0 & $\begin{array}{l}\text { Absolute difference of early-stopping metric } \\
\text { that will be taken into account as an improvement. }\end{array}$ \\
\hline max_epochs & 100 & Maximum number of epochs for training. \\
\hline max_iteration & $1 \mathrm{e} 6$ & Maximum number of updates for training. \\
\hline valid_metric & bleu/meteor/px & $\begin{array}{l}\text { Validation metric(s) (separated by comma) to be } \\
\text { printed, first being the early-stopping metric. }\end{array}$ \\
\hline valid_start & 1 & Start validation after this number of epochs finished. \\
\hline valid_freq & 0 & $\begin{array}{l}\Theta \text { means validations occur at end of epochs while } \\
\text { an explicit <int> defines the period in terms of updates. }\end{array}$ \\
\hline valid_njobs & 16 & Number of CPUs to use during validation beam-search. \\
\hline valid_beam & 12 & The size of the beam during validation beam-search. \\
\hline valid_save_hyp & False/True & Dumps validation hypotheses to separate text files. \\
\hline disp_freq & 10 & The frequency of logging in terms of updates. \\
\hline save_best_n & 4 & $\begin{array}{l}\text { Save } 4 \text { best models on-disk based on validation metric } \\
\text { for further ensembling. }\end{array}$ \\
\hline \multicolumn{3}{|l|}{ [model] options } \\
\hline weight_init & xavier/he/<float> & $\begin{array}{l}\text { Weight initialization method or a }<\text { float }>\text { to define } \\
\text { the scale of random normal distribution. }\end{array}$ \\
\hline batch_size & 32 & Mini-batch size for training. \\
\hline optimizer & $\begin{array}{l}\text { adam/adadelta/ } \\
\text { sgd/rmsprop }\end{array}$ & Stochastic optimizer to use for training. \\
\hline lrate & None/<float > & $\begin{array}{l}\text { If given, overrides the optimizer default defined } \\
\text { in nmtpy/optimizers.py. }\end{array}$ \\
\hline
\end{tabular}

Table 2. Description of options and their default values: when the number of possible values is finite, the default is written in bold. 
Layers Feed-forward layer, highway layer (Srivastava et al., 2015), Gated Recurrent Unit (GRU) (Chung et al., 2014) Conditional GRU (CGRU) (Firat and Cho, 2016) and Multimodal CGRU (Caglayan et al., 2016a,b) are currently available for architecture design. Layer normalization (Ba et al., 2016), a method that adaptively learns to scale and shift the incoming activations of a neuron is available for GRU and CGRU blocks.

Iteration Parallel and monolingual text iterators with compressed (.gz, .bz2, .xz) file support are available under the names TextIterator and BiTextIterator. Additionally, the multimodal WMTIterator allows using image features and source/target sentences at the same time for multimodal NMT (Section 3.3). An efficient target length based batch sorting is available with the option shuffle_mode:trglen.

Training nmtpy provides Theano implementations of stochastic gradient descent (SGD) and its adaptive variants RMSProp (Tieleman and Hinton, 2012), Adadelta (Zeiler, 2012) and Adam (Kingma and Ba, 2014) to optimize the weights of the trained network. A preliminary support for gradient noise (Neelakantan et al., 2015) is available for Adam. Gradient norm clipping (Pascanu et al., 2013) is enabled by default with a threshold of 5 to avoid exploding gradients. Although the provided architectures all use the cross-entropy objective by their nature, any arbitrary differentiable objective function can be used since the training loop is agnostic to the architecture being trained.

Post-processing All decoded translations will be post-processed if filter option is given in the configuration file. This is useful in the case where one would like to compute automatic metrics on surface forms instead of segmented. Currently available filters are bpe and compound for cleaning subword BPE (Sennrich et al., 2016) and German compound-splitting (Sennrich and Haddow, 2015) respectively.

Metrics nmt-train performs a patience based early-stopping using either validation perplexity or one of the automatic metric wrappers i.e. BLEU (Papineni et al., 2002) or METEOR (Lavie and Agarwal, 2007). These metrics are also available for $n m t$-translate to immediately score the produced hypotheses. Other metrics can be easily added and made available as early-stopping metrics.

\section{Architectures}

\subsection{Neural Machine Translation (NMT)}

The NMT architecture (attention) is based on dl4mt-tutorial which differs from Bahdanau et al. (2014) in the following major aspects: 
- The decoder is CGRU (Firat and Cho, 2016) which consists of two GRU interleaved with attention mechanism,

- The hidden state of the decoder is initialized with a non-linear transformation applied to mean bi-directional encoder state instead of last one,

- Maxout (Goodfellow et al., 2013) layer before the softmax operation is removed.

\begin{tabular}{|c|c|c|}
\hline Option & Value(s) (default) & Description \\
\hline init_cgru & zero (text) & $\begin{array}{l}\text { Initializes CGRU with zero instead of mean encoder state } \\
\text { (García-Martínez et al., 2017). }\end{array}$ \\
\hline tied_emb & 2way/3way (False) & $\begin{array}{l}\text { Allows } 2 \text { way and } 3 \text { way sharing of embeddings in the network } \\
\text { (Inan et al., 2016, Iress and wrolf, 2010). }\end{array}$ \\
\hline shuffle_mode & simple (trglen) & Switch between simple and target-length ordered shuffling. \\
\hline layer_norm & bool (False) & Enable/disable layer normalization for GRU encoder. \\
\hline simple_output & bool (False) & $\begin{array}{l}\text { Condition target probability only on decoder's hidden state } \\
\text { (García-Martínez et al., 2017). }\end{array}$ \\
\hline n_enc_layers & $\operatorname{int}(1)$ & $\begin{array}{l}\text { Number of unidirectional encedems to stack on top } \\
\text { of the bi-directional encoder. }\end{array}$ \\
\hline emb_dropout & float $(0)$ & Rate of dropout applied on source embeddings. \\
\hline ctx_dropout & float $(0)$ & Rate of dropout applied on source encoder states. \\
\hline out_dropout & float $(0)$ & Rate of dropout applied on pre-softmax activations. \\
\hline
\end{tabular}

Table 3. Description of configuration options for the NMT architecture.

The final NMT architecture offers many new options which are shortly explained in Table 3 . We also provide a set of auxiliary tools which are useful for pre-processing and post-training tasks (Table $\underline{4}$ ).

\begin{aligned} & \hline Tool Description \\ & \hline nmt-bpe-* Clone of subword utilities for BPE processing (Sennrich et al., 2016). \\ & nmt-build-dict Generates .pkl vocabulary files from corpora prior to training. \\ & nmt-rescore Rescores n-best hypotheses with single/ensemble of models on GPU. \\ & nmt-coco-metrics Computes several metrics using MSCOCO evaluation tools (Chen et al., 2015). \\ & nmt-extract Extracts and saves weights from a trained model instance. \\ & \hline\end{aligned}

Table 4. Brief descriptions of helper NMT tools.

\subsection{Factored NMT (FNMT)}

Factored NMT (FNMT) is an extension of NMT which generates two output symbols (García-Martínez et al., 2016). In contrast to multi-task architectures, FNMT outputs share the same recurrence and output symbols are generated in a synchronous 
fashion. Two variants which differ in how they handle the output layer are currently available: (attention_factors) where the lemma and factor embeddings are concatenated to form a single feedback embedding and (attention_factors_seplogits) where the output path for lemmas and factors are kept separate with different presoftmax transformations applied for specialization.

\subsection{Multimodal NMT (MNMT)}

We provide several multimodal architectures where the probability of a target word is estimated given source sentence representations and visual features: (1) Fusion architectures (Caglayan et al., 2016a,b) extend monomodal CGRU into a multimodal one where a multimodal attention is applied over textual and visual features, (2) MNMT architectures based on global features make use of fixed-width visual features to ground NMT with visual informations (Caglayan et al., 2017).

\subsection{Other}

- A GRU-based reimplementation (img2txt) of Show, Attend and Tell image captioning architecture (Xu et al., 2015),

- A GRU-based language model architecture (rnnlm) to train recurrent language models. nmt-test-lm is the inference utility for perplexity computation of a corpus using a trained checkpoint.

\section{Results}

\begin{tabular}{rlr}
\hline System & MMT & Test2017 Meteor (Rank) \\
\hline NMT & En $\rightarrow$ De & $53.8(\# 3)$ \\
MNMT & En $\rightarrow$ De & $54.0(\# 1)$ \\
\hline NMT & En $\rightarrow$ Fr & $70.1(\# 4)$ \\
MNMT & En $\rightarrow$ Fr & $72.1(\# 1)$ \\
\hline System & News & Test2017 BLEU \\
\hline NMT-UEDIN (Winner) & En $\rightarrow$ Tr & 16.5 \\
NMT-Ours (Post-deadline) & En $\rightarrow$ Tr & 18.1 \\
\hline FNMT & En $\rightarrow$ Lv & 16.2 \\
\hline FNMT & En $\rightarrow$ Cs & 19.9 \\
\hline
\end{tabular}

Table 5. Ensembling scores for LIUM's WMT17 MMT and News Translation submissions. 


\begin{tabular}{rcc}
\hline System & Test2017 BLEU & Test2017 METEOR \\
\hline Nmtpy & $30.8 \pm 1.0$ & $51.6 \pm 0.5$ \\
Nematus & 31.6 & 50.6 \\
\hline
\end{tabular}

Table 6. Mean/std. deviation of 5 Nmtpy runs vs 1 Nematus run for WMT17 MMT En $\rightarrow$ De.

We present our submitted nmtpy systems for Multimodal Translation (MMT) and News Translation tasks of WMT17 (Table 5). For MMT, state-of-the-art results are obtained by our systems (Caglayan et al., 2017) 6 in both $\mathrm{En} \rightarrow$ De and $\mathrm{En} \rightarrow \mathrm{Fr}$ tracks (Elliott et al., 2017). In the context of news translation task, our post-deadline En $\rightarrow \mathrm{Tr}$ NMT system (García-Martínez et al., 2017) surpassed the official winner by 1.6 BLEU.

We also trained a monomodal NMT for WMT17 MMT En $\rightarrow$ De track with Nematus using hyper-parameters very similar to our submitted NMT architecture and found that the results are comparable for BLEU and slightly better for nmtpy in terms of METEOR (Table 6).

\section{Conclusion}

We have presented nmtpy, an open-source sequence-to-sequence framework based on dl4mt-tutorial and refined in many ways to ease the task of integrating new architectures. The toolkit has been internally used in our team for tasks ranging from monomodal, multimodal and factored NMT to image captioning and language modeling to achieve top-ranked campaign results and state-of-the-art performance.

\section{Acknowledgements}

This work was supported by the French National Research Agency (ANR) through the CHIST-ERA M2CR project, under the contract number ANR-15-CHR2-0006-01-.

\section{Bibliography}

Abadi, Martín, Ashish Agarwal, Paul Barham, Eugene Brevdo, Zhifeng Chen, Craig Citro, Greg S Corrado, Andy Davis, Jeffrey Dean, Matthieu Devin, et al. Tensorflow: Large-scale machine learning on heterogeneous distributed systems. arXiv preprint arXiv:1603.04467, 2016.

Ba, Jimmy Lei, Jamie Ryan Kiros, and Geoffrey E Hinton. Layer normalization. arXiv preprint arXiv:1607.06450, 2016. URL http://arxiv.org/abs/1607.06450.

\footnotetext{
${ }^{6}$ http://github. com/lium- lst/wmt17-mmt

${ }^{7}$ http://m2cr. univ- lemans.fr
} 
Bahdanau, Dzmitry, Kyunghyun Cho, and Yoshua Bengio. Neural Machine Translation by Jointly Learning to Align and Translate. CoRR, abs/1409.0473, 2014. URL http://arxiv. org/abs/1409.0473.

Caglayan, Ozan, Walid Aransa, Yaxing Wang, Marc Masana, Mercedes García-Martínez, Fethi Bougares, Loïc Barrault, and Joost van de Weijer. Does Multimodality Help Human and Machine for Translation and Image Captioning? In Proceedings of the First Conference on Machine Translation, pages 627-633, Berlin, Germany, August 2016a. Association for Computational Linguistics. URL http://www. aclweb. org/anthology/W/W16/W16-2358. pdf.

Caglayan, Ozan, Loïc Barrault, and Fethi Bougares. Multimodal Attention for Neural Machine Translation. arXiv preprint arXiv:1609.03976, 2016b. URL http://arxiv.org/abs/ 1609.03976.

Caglayan, Ozan, Walid Aransa, Adrien Bardet, Mercedes García-Martínez, Fethi Bougares, Loïc Barrault, Marc Masana, Luis Herranz, and Joost van de Weijer. LIUM-CVC Submissions for WMT17 Multimodal Translation Task. In Proceedings of the Second Conference on Machine Translation, Copenhagen, Denmark, September 2017.

Chen, Xinlei, Hao Fang, Tsung-Yi Lin, Ramakrishna Vedantam, Saurabh Gupta, Piotr Dollár, and C Lawrence Zitnick. Microsoft COCO captions: Data collection and evaluation server. arXiv preprint arXiv:1504.00325, 2015.

Chung, Junyoung, Çaglar Gülçehre, KyungHyun Cho, and Yoshua Bengio. Empirical Evaluation of Gated Recurrent Neural Networks on Sequence Modeling. CoRR, abs/1412.3555, 2014. URL http://arxiv.org/abs/1412.3555.

Elliott, Desmond, Stella Frank, Loïc Barrault, Fethi Bougares, and Lucia Specia. Findings of the Second Shared Task on Multimodal Machine Translation and Multilingual Image Description. In Proceedings of the Second Conference on Machine Translation, Copenhagen, Denmark, September 2017.

Firat, Orhan and Kyunghyun Cho. Conditional Gated Recurrent Unit with Attention Mechanism. github.com/nyu-dl/dl4mt - tutorial/blob/master/docs/cgru . pdf, 2016.

García-Martínez, Mercedes, Loïc Barrault, and Fethi Bougares. Factored Neural Machine Translation Architectures. In Proceedings of the International Workshop on Spoken Language Translation, IWSLT'16, Seattle, USA, 2016. URL http://workshop2016.iwslt.org/downloads/ IWSLT\_2016\_paper\_2.pdf.

García-Martínez, Mercedes, Ozan Caglayan, Walid Aransa, Adrien Bardet, Fethi Bougares, and Loïc Barrault. LIUM Machine Translation Systems for WMT17 News Translation Task. In Proceedings of the Second Conference on Machine Translation, Copenhagen, Denmark, September 2017.

Glorot, Xavier and Yoshua Bengio. Understanding the difficulty of training deep feedforward neural networks. In Proceedings of the Thirteenth International Conference on Artificial Intelligence and Statistics, volume 9 of Proceedings of Machine Learning Research, pages 249-256. PMLR, 13-15 May 2010. URL http://proceedings.ml r.press/v9/glorot10a.html.

Goodfellow, Ian, David Warde-Farley, Mehdi Mirza, Aaron Courville, and Yoshua Bengio. Maxout Networks. In Dasgupta, Sanjoy and David McAllester, editors, Proceedings of the 30th International Conference on Machine Learning, volume 28 of Proceedings of Machine 
Learning Research, pages 1319-1327, Atlanta, Georgia, USA, 17-19 Jun 2013. PMLR. URL http://proceedings.mlr.press/v28/goodfellow13.html.

He, Kaiming, Xiangyu Zhang, Shaoqing Ren, and Jian Sun. Delving Deep into Rectifiers: Surpassing Human-Level Performance on ImageNet Classification. In Computer Vision (ICCV), 2015 IEEE International Conference on, pages 1026-1034. IEEE, 2015.

Helcl, Jindřich and Jindřich Libovický. Neural Monkey: An Open-source Tool for Sequence Learning. The Prague Bulletin of Mathematical Linguistics, (107):5-17, 2017. ISSN 0032-6585. doi: 10.1515/pralin-2017-0001. URL http://ufal.mff.cuni.cz/pbml/107/ art-helcl-libovicky. pdf.

Inan, Hakan, Khashayar Khosravi, and Richard Socher. Tying Word Vectors and Word Classifiers: A Loss Framework for Language Modeling. arXiv preprint arXiv:1611.01462, 2016.

Kingma, Diederik and Jimmy Ba. Adam: A method for stochastic optimization. arXiv preprint arXiv:1412.6980, 2014. URL http://arxiv.org/abs/1412.6980.

Klein, G., Y. Kim, Y. Deng, J. Senellart, and A. M. Rush. OpenNMT: Open-Source Toolkit for Neural Machine Translation. ArXiv e-prints, 2017.

Lavie, Alon and Abhaya Agarwal. Meteor: An Automatic Metric for MT Evaluation with High Levels of Correlation with Human Judgments. In Proceedings of the Second Workshop on Statistical Machine Translation, StatMT '07, pages 228-231, Stroudsburg, PA, USA, 2007. Association for Computational Linguistics. URL http://dl . acm.org/citation. cfm?id=1626355. 1626389.

Neelakantan, Arvind, Luke Vilnis, Quoc V Le, Ilya Sutskever, Lukasz Kaiser, Karol Kurach, and James Martens. Adding gradient noise improves learning for very deep networks. arXiv preprint arXiv:1511.06807, 2015. URL http://arxiv.org/abs/1511.06807.

Papineni, Kishore, Salim Roukos, Todd Ward, and Wei-Jing Zhu. BLEU: A Method for Automatic Evaluation of Machine Translation. In Proceedings of the 40th Annual Meeting on Association for Computational Linguistics, ACL '02, pages 311-318, Stroudsburg, PA, USA, 2002. Association for Computational Linguistics. doi: 10.3115/1073083.1073135. URL http://dx.doi.org/10.3115/1073083.1073135.

Pascanu, Razvan, Tomas Mikolov, and Yoshua Bengio. On the Difficulty of Training Recurrent Neural Networks. In Proceedings of the 30th International Conference on International Conference on Machine Learning - Volume 28, ICML'13, pages III-1310-III-1318. JMLR.org, 2013. URL http://dl.acm.org/citation. cfm?id=3042817. 3043083.

Press, Ofir and Lior Wolf. Using the output embedding to improve language models. arXiv preprint arXiv:1608.05859, 2016.

Saxe, Andrew M, James L McClelland, and Surya Ganguli. Exact solutions to the nonlinear dynamics of learning in deep linear neural networks. arXiv preprint arXiv:1312.6120, 2013.

Sennrich, Rico and Barry Haddow. A Joint Dependency Model of Morphological and Syntactic Structure for Statistical Machine Translation. In Proceedings of the 2015 Conference on Empirical Methods in Natural Language Processing, pages 114-121. Association for Computational Linguistics, 2015. 
Sennrich, Rico, Barry Haddow, and Alexandra Birch. Neural Machine Translation of Rare Words with Subword Units. In Proceedings of the 54th Annual Meeting of the Association for Computational Linguistics (Volume 1: Long Papers), pages 1715-1725, Berlin, Germany, August 2016. Association for Computational Linguistics. URL http://www. aclweb. org/anthology/ P16 - 1162.

Sennrich, Rico, Orhan Firat, Kyunghyun Cho, Alexandra Birch-Mayne, Barry Haddow, Julian Hitschler, Marcin Junczys-Dowmunt, Samuel Läubli, Antonio Miceli Barone, Jozef Mokry, and Maria Nadejde. Nematus: a Toolkit for Neural Machine Translation, pages 65-68. Association for Computational Linguistics (ACL), 4 2017. ISBN 978-1-945626-34-0.

Srivastava, Nitish, Geoffrey Hinton, Alex Krizhevsky, Ilya Sutskever, and Ruslan Salakhutdinov. Dropout: A Simple Way to Prevent Neural Networks from Overfitting. J. Mach. Learn. Res., 15(1):1929-1958, Jan. 2014. ISSN 1532-4435. URL http://dl . acm. org/citation. cfm? id $=2627435.2670313$.

Srivastava, Rupesh Kumar, Klaus Greff, and Jürgen Schmidhuber. Highway networks. arXiv preprint arXiv:1505.00387, 2015.

Theano Development Team. Theano: A Python framework for fast computation of mathematical expressions. arXiv e-prints, abs/1605.02688, 2016. URL http://arxiv.org/abs/1605. 02688.

Tieleman, Tijmen and Geoffrey Hinton. Lecture 6.5-rmsprop: Divide the gradient by a running average of its recent magnitude. COURSERA: Neural networks for machine learning, 4(2), 2012.

$\mathrm{Xu}$, Kelvin, Jimmy Ba, Ryan Kiros, Kyunghyun Cho, Aaron Courville, Ruslan Salakhudinov, Rich Zemel, and Yoshua Bengio. Show, Attend and Tell: Neural Image Caption Generation with Visual Attention. In Proceedings of the 32nd International Conference on Machine Learning (ICML-15), pages 2048-2057. JMLR Workshop and Conference Proceedings, 2015. URL http://jmlr.org/proceedings/papers/v37/xuc15.pdf.

Zeiler, Matthew D. ADADELTA: an adaptive learning rate method. arXiv preprint arXiv:1212.5701, 2012. 


\title{
Appendix A: Example NMT Configuration
}

\author{
\# Options in this section are consumed by nmt-train \\ [training] \\ model_type: attention \# Model type without.py \\ patience: 20 \# early-stopping patience \\ valid_freq: $1000 \quad$ \# Compute metrics each 1000 updates \\ valid_metric: meteor \# Use meteor during validations \\ valid_start: 2 \# Start validations after 2nd epoch \\ valid_beam: 3 \# Decode with beam size 3 \\ valid_njobs: 16 \# Use 16 processes for beam-search \\ valid_save_hyp: True \# Save validation hypotheses \\ decay_c: 1e-5 \# L2 regularization factor \\ clip_c: 5 \# Gradient clip threshold \\ seed: 1235 \# Seed for numpy and Theano RNG \\ save_best_n: 2 \# Keep 2 best models on-disk \\ device id: auto \# Pick 1st available GPU \\ max epochs: 100
}

\# Options in this section are passed to model instance

[model]

tied_emb: 2way

layer_norm: True

\# weight-tying mode (False, 2way, 3way)

shuffle mode: trglen

\# Layer norm in GRU encoder

filter: bpe

n_words_src: 0

\# Shuffled/length-ordered batches

\# post-processing filter(s)

\# limit src vocab if $>0$

\# limit trg vocab if $>0$

save_path: $\sim /$ models

\# Where to store checkpoints

rnn dim: 100

\# Encoder and decoder RNN dim

embedding_dim: 100

\# All embedding dim

weight init: xavier

batch size: 32

optimizer: adam

lrate: 0.0004

emb_dropout: 0.2

\# Set dropout rates

ctx_dropout: 0.4

out_dropout: 0.4

\# Vocabulary paths produced by nmt-build-dict

[model.dicts]

src: /data/train.norm. max50.tok. lc.bpe.en.pkl

trg: $\sim /$ data/train.norm. max50.tok.lc.bpe.de.pkl

\# Training and validation data

[model.data]

train_src : $\sim /$ data/train.norm.max50.tok.lc.bpe.en

train trg : $\sim /$ data/train.norm.max50.tok.lc.bpe.de

valid_src : /data/val.norm.tok.lc.bpe.en

valid_trg : /data/val.norm.tok.lc.bpe.de \# BPE refs for validation perplexity

valid_trg_orig: /data/val.norm.tok.lc.de \# non-BPE refs for correct metric computation 


\section{Appendix B: Installation}

nmtpy requires a Python 3 environment with NumPy and Theano v0.9 installed. A Java runtime ( $j$ ava should be in the PATH) is also needed by the METEOR implementation. You can run the below commands in the order they are given to install nmtpy into your Python environment:

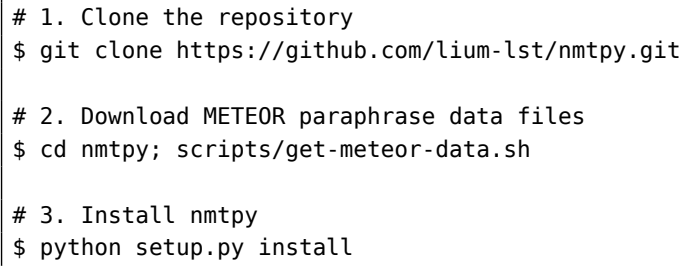

Note that once you installed nmtpy with python setup. py install, any modifications to the source tree will not be visible until nmtpy is reinstalled. If you would like to avoid this because you are constantly modifying the source code (for adding new architectures, iterators, features), you can replace the last command above by python setup. py develop. This tells the Python interpreter to directly use nmtpy from the GIT folder. The final alternative is to copy scripts/snaprun into your \$PATH, modify it to point to your GIT folder and launch training using it as in below:

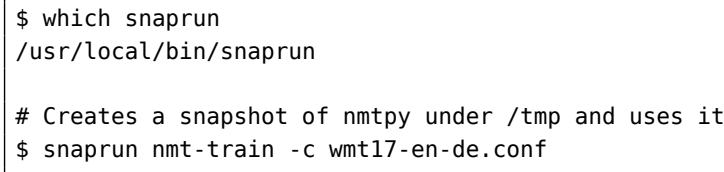

Performance In order to get the best speed in terms of training and beam-search, we recommend using a recent version of CUDA, CuDNN and a NumPy linked against Intel MKL $\stackrel{8}{-}$ or OpenBLAS.

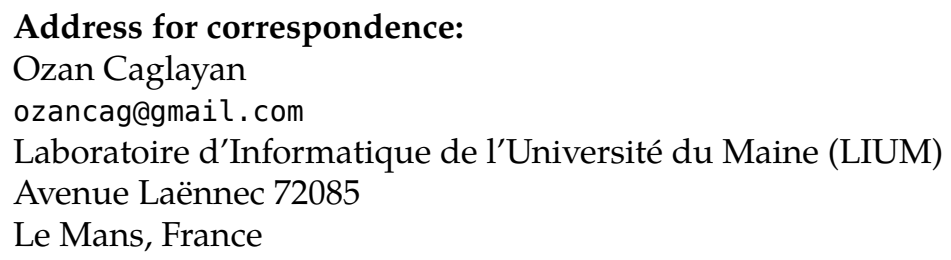

${ }^{8}$ Anaconda Python distribution is a good option which already ships an MKL-enabled NumPy. 